\title{
A Combinatorial Interpretation of the Free Fermion Condition of the Six-Vertex Model
}

\author{
R. Brak and A. Owczarek, \\ Department of Mathematics and Statistics, \\ The University of Melbourne, \\ Parkville, Victoria 3052, Australia
}

10th March, 1999

\begin{abstract}
The free fermion condition of the six-vertex model provides a 5 parameter submanifold on which the Bethe Ansatz equations for the wavenumbers that enter into the eigenfunctions of the transfer matrices of the model decouple, hence allowing explicit solutions. Such conditions arose originally in early field-theoretic S-matrix approaches. Here we provide a combinatorial explanation for the condition in terms of a generalised Gessel-Viennot involution. By doing so we extend the use of the Gessel-Viennot theorem, originally devised for non-intersecting walks only, to a special weighted type of intersecting walk, and hence express the partition function of $N$ such walks starting and finishing at fixed endpoints in terms of the single walk partition functions.
\end{abstract}

PACS numbers: 05.50.+q, 05.70.fh, 61.41.+e

Key words: Lattice Paths, Six-vertex model, osculating paths, Gessel-Viennot theorem, free fermion condition.

*email: r.brak, aleks@ms.unimelb.edu.au 


\section{Introduction}

There has been a steady stream of interest in the statistical mechanics of directed walk problems because of their connections to the physics of polymers and domain walls [1]. Since the popularisation of the field in the seminal article by Fisher [1], vicious walkers, in particular, also known as non-intersecting walks, on two-dimensional (directed) lattices have been the subject of much work [2, 3, 4, 5, 6, 7]. In the field of combinatorics a very general methodology, useful for any directed graph and based on a involution, has been developed by Gessel and Viennot [8, 9] following the work of Lindström [10], and Karlin and McGregor [11, which expresses the generating function of configurations of $N$ walks as the value of a determinant of single walk generating functions. In the most general setup an arbitrary inhomogeneous weight may be associated with each occupied edge of the lattice.

On the other hand it has been well known for a long time [12, 7] that the square lattice six-vertex model can be mapped onto a problem of interacting (intersecting) directed walks on that lattice (see figure 1). Let us call the six weights of that model $\left\{w_{1}, w_{2}, w_{3}, w_{4}, w_{5}, w_{6}\right\}$ : see figure 1. We can consider $w_{1}=1$ without loss of generality. In order to calculate the partition function of fixed numbers of walks one needs to consider a particular invariant sub-space of the associated transfer matrix, the diagonalisation of which involves the famous Bethe Ansatz trial solution: The Bethe Ansatz is a guess for the eigenvectors of the transfer matrix and involves a sum over a set of plane wave forms. For a problem of $N$ walks the Bethe Ansatz involves $N$ wavenumbers which are chosen from the solutions of a set of $N$ non-linear coupled polynomial equations. To calculate the walk partition function one needs to find, and be able to sum over, all the eigenvalues and eigenvectors explicitly.

Recently [13] it has been shown that if one rather considers the combinatorialist's problem of $N$ vicious walkers with weights associated with edges, rather than with vertices, this can be solved using the transfer matrix/Bethe Ansatz approach in a completely rigorous fashion. Here the Bethe Ansatz equations for $N$ walks decouple and the solution of the $N$ walk problem is given by the Gessel-Viennot determinant of single walk generating functions. The walk problem using edge weights is equivalent to a restricted vertex model (or visa versa) where the vertex weights must satisfy the equations

$$
w_{3} w_{4}=w_{5} w_{6}
$$

and

$$
w_{2}=0
$$


The second condition (1.2) merely expresses the fact that the weight associated with the meeting of two walks is set to zero since vicious walkers are being considered. The associated vertex model with only this second condition necessarily holding is often referred to as the five vertex model.

Of central importance here is that taken together the two conditions, (1.1) and (1.2), imply that the less restrictive free-fermion condition of the six-vertex model, which occurs when the vertex weights satisfy the equation

$$
w_{1} w_{2}=w_{5} w_{6}-w_{3} w_{4}
$$

is then automatically satisfied. Hence the edge weight model is equivalent to the freefermion case of the five-vertex model. This is not surprising since the free-fermion condition (1.3) is precisely the general condition needed to achieve the decoupling of the Bethe Ansatz equations in the solution of the six-vertex model.

This raises the question of whether the Gessel-Viennot methodology can be adapted to the 'free-fermion' case (i.e. equation (1.3) is satisfied), of 'six-vertex' or 'osculating' walks - here the walks are allowed to intersect but not share edges i.e. site-only intersecting.
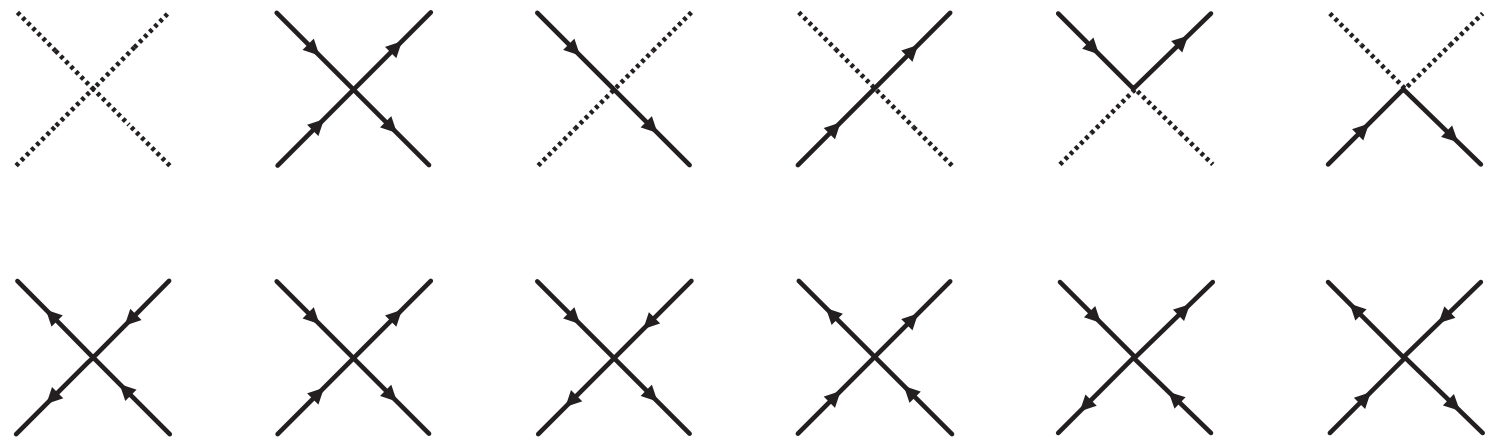

$w_{1}$

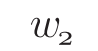

$w_{3}$

$w_{4}$

$w_{5}$

$w_{6}$

Figure 1: At the top are the six possible path configurations at a vertex of the lattice. Below each of these is the associated arrow configuration of the six-vertex model, while below that is the six weights we associate with each of those configurations.

In this paper we demonstrate that free-fermion osculating walks can indeed be counted with a generalisation of the Gessel-Viennot methodology and we hence explicitly calculate their generating function. Because the Gessel-Viennot involution is involved the result is again a determinant of single walk generating functions. We restrict our discussion to the square lattice but the ideas can be easily generalised to any directed (acyclic) graph. In order to understand the combinatorial interpretation of the osculating free-fermion walks we briefly discuss the case of non-intersecting walks for those unfamiliar with the GesselViennot method. For the case of non-intersecting walks the Gessel-Viennot theorem gives 


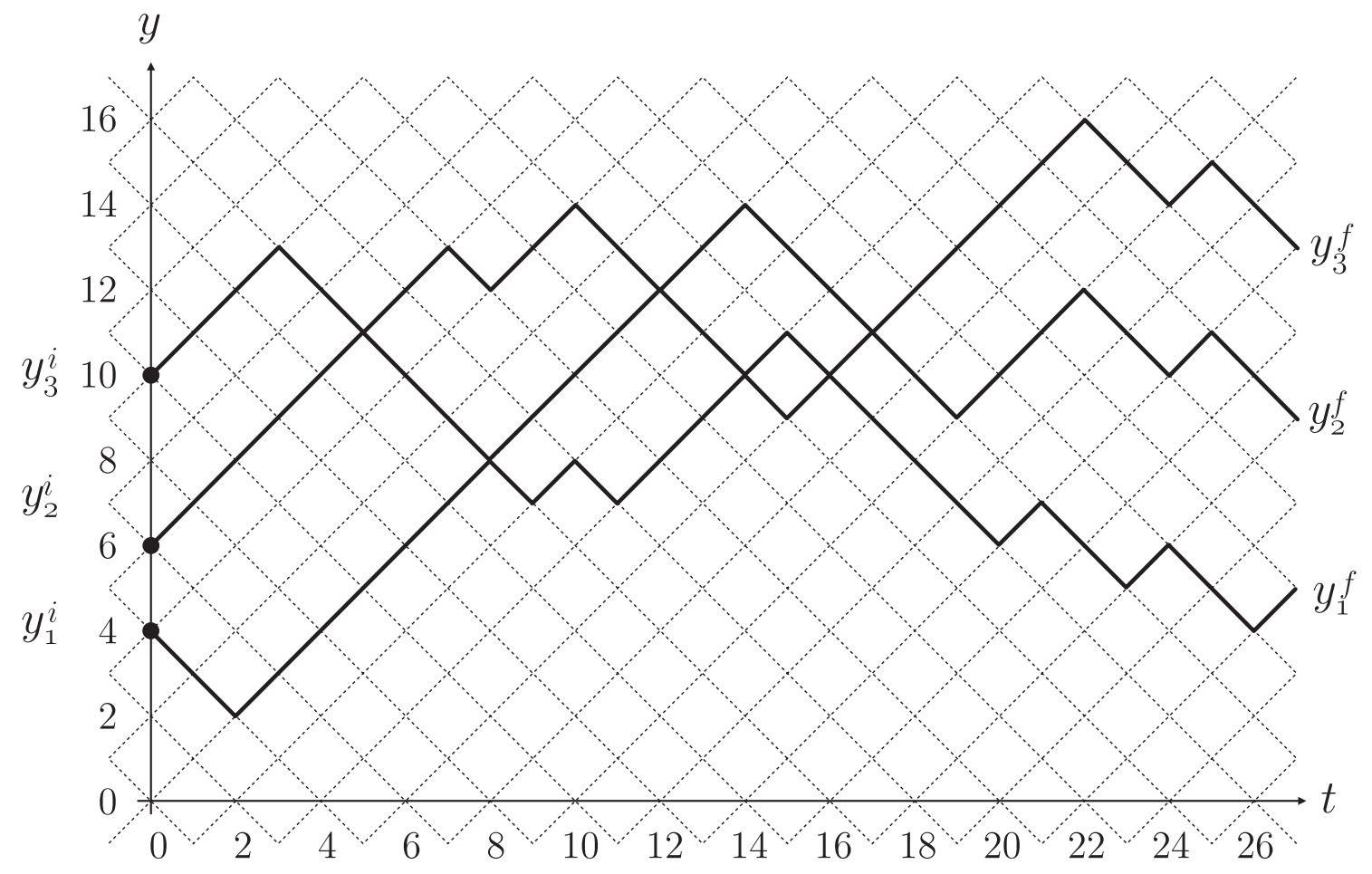

Figure 2: An example of three osculating paths.

the generating function of $N$ such paths starting and ending at some fixed sets of sites as the determinant of the generating functions of independent-single-walks where the walks start and end at all permutations of those endpoints. That is, the generating function, $\mathcal{N}_{t}^{(N)}\left(\mathbf{y}^{i} \rightarrow \mathbf{y}^{f}\right)$, for $N$ non-intersecting walk configurations where the $j^{\text {th }}$ walk starts at $y_{j}^{i}$ and arrives at $y_{j}^{f}$ after $t$ steps is given by the following determinant:

$$
\mathcal{N}_{t}^{(N)}\left(\mathbf{y}^{i} \rightarrow \mathbf{y}^{f}\right)=\operatorname{det}\left\|\mathcal{N}_{t}^{(1)}\left(y_{\alpha}^{i} \rightarrow y_{\beta}^{f}\right)\right\|_{\alpha, \beta=1 \ldots N} .
$$

where $\mathbf{y}^{i}=\left(y_{1}^{i}, \ldots, y_{N}^{i}\right)$ and $\mathbf{y}^{f}=\left(y_{1}^{f}, \ldots, y_{N}^{f}\right)$. Hence the generating function of $N$ walks is given as the signed sum over products of $N$ single walk generating functions where the sign of the contribution is signature of the permutation of the endpoints. This signed sum is interpreted as a signed sum over the elements of a "signed" set, $\Omega$, the elements of which are configurations of $N$, possibly intersecting, walks,

$$
\mathcal{F}_{t}^{(N)}\left(\mathbf{y}^{i} \rightarrow \mathbf{y}^{f}\right)=\sum_{\mathbf{X} \in \Omega} \epsilon_{\mathbf{X}} \prod_{\alpha=1}^{N} \mathcal{M}_{t}^{(1)}\left(X_{\alpha}\right) .
$$

where $X_{\alpha}$ is a single walk from $y_{\alpha}^{i} \rightarrow y_{\beta}^{f}, \epsilon_{\mathbf{X}}$ is the sign of the configuration and $\mathcal{M}_{t}^{(1)}\left(X_{\alpha}\right)$ the weight of the a particular configuration of a single walk - see section $⿴$ for more precise definitions of the terms referred to in this section. Note that the $N$ single walks in elements of $\Omega$ may be edge as well as site intersecting, as they are completely independent of each other. 
The method introduced by Gessel and Viennot [8, 9] shows that pairs of opposite signed terms of (1.5) corresponding to configurations with at least one intersection cancel leaving only positively signed terms corresponding to non-intersecting configurations precisely the sum required to give $\mathcal{N}_{t}^{(N)}\left(\mathbf{y}^{i} \rightarrow \mathbf{y}^{f}\right)$. This pairing rule is an "involution".

In order to interpret the free-fermion condition for osculating or six-vertex walks we apply the same type of methodology but now require an extended pairing rule, one for which the pairs of terms in the signed sum do not necessarily cancel out. We now consider three different disjoint subsets of $\Omega$ : $\Omega^{n}$ the subset containing configurations that do not intersect at all, $\Omega^{s}$ the subset containing configurations that only share sites (and not edges) and $\Omega^{e}$ the subset containing configurations that share at least one edge.

The involution now pairs oppositely signed terms from $\Omega^{e}$ which cancel out, but the pairs of oppositely signed terms from the subset $\Omega^{s}$ have different weights and do not cancel out, rather the weight difference is precisely given by the free-fermion condition (1.3). This idea is expressed schematically by the case for (example) paths in the subset $\Omega^{s}$ as

$$
\mathcal{P}(\widetilde{\Upsilon})-\mathcal{P}\left(\Upsilon^{\prime}\right)=\mathcal{W}(\widetilde{\Upsilon})
$$

and for walks in the subset $\Omega^{e}$ as

$$
\mathcal{P}(\text { ๙ })-\mathcal{P}(\nwarrow)=0
$$

where $\mathcal{P}(X)$ is the product of the vertex weights of the two single paths of the configuration $X$, whilst $\mathcal{W}(X)$ is the vertex weight of the paths, $X$, taken as a whole.

Thus the free fermion condition arises as a natural consequence of summing over a signed set of $N$-walk configurations each of whose weight is a product of single path vertex weights. The intersecting configurations in the signed set which do not cancel combine to create the correct vertex weight for the $N$-walk configuration.

\section{Free Fermion walk generating function}

Definitions and Notations We will consider walks on the directed square lattice, $\mathcal{L}$ rotated through $45^{\circ}$. Each vertex of the lattice is labelled by the "time" coordinate, $t$ and a height $y$ and represented by the pair $(t, y)$ or function $y(t)$ - see figure 2. An $N$-vertex is an $N$-tuple of vertices of $\mathcal{L}$ each of has the same time coordinate and is represented by the $N$-tuple of their height coordinates, $\mathbf{y}=\left(y_{1}, \ldots, y_{N}\right)$. An $N$-vertex $\mathbf{y}$ is non-intersecting if $y_{\beta} \neq y_{\alpha}, \alpha, \beta=1, \ldots, N, \alpha \neq \beta$. A walk of length $t, X\left(y_{\alpha}, y_{\beta}\right)=$ $y(0) y(1) y(2) \ldots y(t)$, where $y(0)=y_{\alpha}$ and $y(t)=y_{\beta}$, is a sequence of $t$ adjacent edges 
from vertex $y(0)$ to vertex $y(t)$. An $N$-walk, $\mathbf{X}\left(\mathbf{y}^{i}, \mathbf{y}^{f}\right)$ is an $N$-tuple of equal length walks, $\left(X_{1}\left(y_{1}^{i}, y_{1}^{f}\right), \ldots, X_{N}\left(y_{N}^{i}, y_{N}^{f}\right)\right)$, with $\mathbf{y}^{i}$ and $\mathbf{y}^{f}$ non-intersecting.

An osculation between walks $X_{\alpha}$ and $X_{\beta}$ at time $s$ occurs if $y_{\alpha}(s)=y_{\beta}(s)$, and either $y_{\alpha}(s-1)<y_{\beta}(s-1)$ and $y_{\alpha}(s+1)<y_{\beta}(s+1)$ or $y_{\alpha}(s-1)>y_{\beta}(s-1)$ and $y_{\alpha}(s+1)>y_{\beta}(s+1)$ occur i.e the configuration $\underset{\sim}{\sim}$ appears. Paths $X_{\alpha}$ and $X_{\beta}$ switch at time $s$ if $y_{\alpha}(s)=y_{\beta}(s)$, and either $y_{\alpha}(s-1)<y_{\beta}(s-1)$ and $y_{\alpha}(s+1)>y_{\beta}(s+1)$ or $y_{\alpha}(s-1)>y_{\beta}(s-1)$ and $y_{\alpha}(s+1)<y_{\beta}(s+1)$ occur i.e the configuration $\not K$ appears. Since the initial and final $N$-vertices are non-intersecting we do not define any osculations or switches associated with these vertices. A pair of walks is osculating if all common vertices are osculations - see figure 2. An $N$-walk $\mathbf{X}$ is osculating if all the vertices in common with any of the walks form osculations.

Let $X_{\alpha}=y_{\alpha}(0) y_{\alpha}(1) y_{\alpha}(2) \ldots y_{\alpha}(t)$ and $X_{\beta}=y_{\beta}(0) y_{\beta}(1) y_{\beta}(2) \ldots y_{\beta}(t)$ intersect at time $s$ i.e $y_{\alpha}(s)=y_{\beta}(s)$, then the pair $X_{\alpha}$ and $X_{\beta}$ are exchanged at time $s$ if

$$
\begin{aligned}
& X_{\alpha} \rightarrow X_{\alpha}^{\prime}=y_{\alpha}(0) \ldots y_{\alpha}(s) y_{\beta}(s+1) \ldots y_{\beta}(t) \\
& X_{\beta} \rightarrow X_{\beta}^{\prime}=y_{\beta}(0) \ldots y_{\beta}(s) y_{\alpha}(s+1) \ldots y_{\alpha}(t)
\end{aligned}
$$

Define $[N]=\{1, \ldots, N\}$. Let $P_{N}$ be the set of permutations of $[N]$, then for $\sigma=$ $\left(\sigma_{1}, \ldots, \sigma_{N}\right) \in P_{N}$, and $\mathbf{y}=\left(y_{1}, \ldots, y_{N}\right)$ an $N$-vertex, then $\sigma(\mathbf{y})=\left(y_{\sigma_{1}}, \ldots, y_{\sigma_{N}}\right)$. The signature of a permutation is denoted $\epsilon_{\sigma}$.

We will associate weights with the walks on the lattice as follows. Associate a set of six vertex weights,

$$
\mathcal{V}(v)=\left\{w_{1}(v), \ldots, w_{6}(v)\right\}
$$

with each vertex $v \in \mathcal{L}$. Without loss of generality we only consider the situation where five of the weights are not equal to one. The vertex weight, $\mathcal{W}(\mathbf{X})$ of a particular $N$-walk, $\mathbf{X}$, is the product of the vertex weights of all the vertices of $\mathcal{L}$ that are traversed by the walks of $N$-walk. For each vertex traversed by the $N$-walk, only one of the six possible vertex weights associated with the vertex of $\mathcal{L}$ is used. Which of the six possible weight used depends on which of the four edges adjacent to the particular vertex are traversed by the $N$-walk as illustrated in figure 1. No vertex weights are associated with the initial and final $N$-vertices of the $N$-walk.

Remark. Note that with the above definition of osculating walks the set includes nonintersecting walks also.

Remark. The vertex weight $\mathcal{W}(\mathbf{X})$ is only defined for osculating paths., i.e if the paths of the $N$-walk do not cross or if $\mathbf{X}$ contains only one path. 
Remark. This way of associating weights with the walks is a generalisation of the situation dealt with by the Gessel-Viennot theorem where the weights are associated with the edges of the graph and are independent of the $N$-walk configuration. The problem of finding the generating function for osculating walks with arbitrary weights requires a rather complicated involution [14, however in the special case where the vertex weights satisfy the free fermion equation the osculating walk generating function can be expressed as a determinant, as we demonstrate here.

Theorem [Free Fermion Walks]. Let $\Omega^{*}$ be the set of all osculating $N$-walks of length $t$ starting at $\mathbf{y}^{i}$ and ending at $\mathbf{y}^{f}$ with $y_{\alpha}^{i}<y_{\alpha+1}^{i}$ and $y_{\alpha}^{f}<y_{\alpha+1}^{f}, \alpha \in[N-1]$. If, for $v \in \mathcal{L}$, the vertex weights satisfy

$$
w_{5}(v) w_{6}(v)-w_{3}(v) w_{4}(v)=w_{2}(v)
$$

then the osculating lattice walk generating function,

$$
\mathcal{F}_{t}^{(N)}\left(\mathbf{y}^{i} \rightarrow \mathbf{y}^{f}\right)=\sum_{\mathbf{X} \in \Omega^{*}} \mathcal{W}(\mathbf{X})
$$

where $\mathcal{W}(\mathbf{X})$ is the vertex weight of the $N$-walk $\mathbf{X}$, is given by

$$
\mathcal{F}_{t}^{(N)}\left(\mathbf{y}^{i} \rightarrow \mathbf{y}^{f}\right)=\left.\operatorname{Det}|| \mathcal{F}_{t}^{(1)}\left(y_{\alpha}^{i} \rightarrow y_{\beta}^{f}\right)\right|_{\alpha, \beta=1 \ldots N}
$$

where $\mathcal{F}_{t}^{(1)}\left(y_{\alpha}^{i} \rightarrow y_{\beta}^{f}\right)$ is the generating function for a single lattice walk from $y_{\alpha}^{i} \rightarrow y_{\beta}^{f}$.

The theorem is proved by an extension of the Gessel-Viennot involution which does not preserve the $N$-walk weight.

Proof. Consider the signed set $\Omega=\Omega^{+} \cup \Omega^{-}, \Omega^{+} \cap \Omega^{-}=\phi$, where $\phi$ is the empty set. The positive and negative sets are

$$
\begin{aligned}
& \Omega^{+}=\left\{\mathbf{X}\left(\mathbf{y}^{i}, \sigma\left(\mathbf{y}^{f}\right)\right) \mid \sigma \in P_{N} \text { and } \epsilon_{\sigma}=+1\right\} \\
& \Omega^{-}=\left\{\mathbf{X}\left(\mathbf{y}^{i}, \sigma\left(\mathbf{y}^{f}\right)\right) \mid \sigma \in P_{N} \text { and } \epsilon_{\sigma}=-1\right\}
\end{aligned}
$$

Let, $\mathbf{X} \in \Omega$, then the sign of $\mathbf{X}$ is defined as

$$
\epsilon_{\mathbf{X}}= \begin{cases}+1 & \text { if } \mathbf{X} \in \Omega^{+} \\ -1 & \text { if } \mathbf{X} \in \Omega^{-}\end{cases}
$$

For the $N$-walks of $\Omega$ we do not use the vertex weight of the $N$-walk as a whole, but rather define a "product" weight, $\mathcal{P}(\mathbf{X})$ in terms of its individual walks. In particular,

$$
\mathcal{P}(\mathbf{X})=\prod_{X_{\alpha} \in \mathbf{X}} \mathcal{W}\left(X_{\alpha}\right)
$$


We construct a sign reversing involution】, $\psi$ on $\Omega$.

Let $\mathbf{X}=\left(X_{1}, \ldots, X_{N}\right) \in \Omega$. The involution is an extension of the Gessel-Viennot involution and splits into three cases,

1. No intersections $\left(\mathbf{X} \in \Omega^{n}\right)$. If none of the walks of $\mathbf{X}$ intersect, then $\psi(\mathbf{X})=\mathbf{X}$.

2. Edge intersections $\left(\mathbf{X} \in \Omega^{e}\right)$. If any of the walks $X_{\alpha} \in \mathbf{X}$ has an edge of $\mathcal{L}$ in common with another walk, $X_{\beta} \in \mathbf{X}$ then let $\alpha$ be the least integer for which a walk $X_{\alpha}$ shares an edge with another walk $X_{\beta}$. Of all the vertices adjacent to the edges in common with $X_{\alpha}$ and $X_{\beta}$ choose the one with the smallest $t$ coordinate and denote it by $v$, then $\mathbf{X}^{\prime}=\psi(\mathbf{X})$ is defined as the $N$-walk obtained by exchanging $X_{\alpha}$ and $X_{\beta}$ at $v$.

3. Vertex only intersections $\left(\mathbf{X} \in \Omega^{s}\right)$. If any of the walks of $\mathbf{X}$ share vertices and none share edges, then let $\alpha$ be the least integer for which a walk $X_{\alpha}$ intersects another walk $X_{\beta}$. Of all the vertices in common with $X_{\alpha}$ and $X_{\beta}$ choose the one with the smallest $t$ coordinate and denote it by $v$, then $\mathbf{X}^{\prime}=\psi(\mathbf{X})$ is defined as the $N$-walk obtained by exchanging $X_{\alpha}$ and $X_{\beta}$ at $v$.

Call the vertex, $v$ at which the involution exchange takes place, the "involution" vertex. The difference in the product weight of $\mathbf{X}$ and $\psi(\mathbf{X})$ is then

$$
\mathcal{P}(\mathbf{X})-\mathcal{P}(\psi(\mathbf{X}))= \begin{cases}\left(w_{5}(v) w_{6}(v)-w_{3}(v) w_{4}(v)\right) \mathcal{P}_{\{v\}}(\mathbf{X}) & \text { if } v \text { is an osculation } \\ \left(w_{3}(v) w_{4}(v)-w_{5}(v) w_{6}(v)\right) \mathcal{P}_{\{v\}}(\mathbf{X}) & \text { if } v \text { is a switch } \\ 0 & \text { otherwise }\end{cases}
$$

where $\mathcal{P}_{\{v\}}(\mathbf{X})$ is the product weight of $\mathbf{X}$ with the contribution of the weight associated with $v$ divided out.

Since $\Omega^{s} \subset \Omega$ is defined as the set of $N$-walks for which the involution vertex $v$ exists and arises from the "vertex only intersections" case of $\psi$, this means that all the $N$-walks in $\Omega^{s}$ have walks which only intersect at vertices - there are no shared edges. Define two $N$-walks to be related, $\mathbf{X} \sim \mathbf{X}^{\prime}$ iff $\mathbf{X}$ can be obtained from $\mathbf{X}^{\prime}$ by the interchange of any number of osculations with switches (or visa versa). This relation is easily seen to be an equivalence relation and hence partitions $\Omega^{s}$ into disjoint subsets, $\hat{\Omega}_{\alpha}^{s}, \alpha \in \mathcal{I}$ where $\mathcal{I}$ is

\footnotetext{
${ }^{1} \mathrm{~A}$ sign reversing involution, $\psi$ is a permutation of $\Omega$ such that $\psi^{2}=$ Identity and it has the property that whenever $\psi(\mathbf{X}) \neq \mathbf{X}$, then $\mathbf{X} \in \Omega^{+}$, if and only if $\psi(\mathbf{X}) \in \Omega^{-}$.
} 
some index set for the partitions. Define the canonical element, $\mathbf{X}^{c_{\alpha}}$ of each partition, $\hat{\Omega}_{\alpha}^{s}$, as the $N$-walk for which all the intersections are osculations. Let $\mathcal{E}\left(\mathbf{X}^{c_{\alpha}}\right)$ be the set of vertices of $\mathbf{X}^{c_{\alpha}} \in \hat{\Omega}_{\alpha}^{s}$ in common with at least two walks of $\mathbf{X}^{c_{\alpha}}$. Note, the cardinality of $\hat{\Omega}_{\alpha}^{s}$ is $2^{\left|\mathcal{E}\left(\mathbf{X}^{c_{\alpha}}\right)\right|}$.

For the $N$-walks in $\hat{\Omega}_{\alpha}^{s}$ we have,

$$
\sum_{\mathbf{X} \in \hat{\Omega}_{\alpha}^{s}} \epsilon_{\mathbf{X}} \mathcal{P}(\mathbf{X})=\mathcal{P}_{\mathcal{E}}\left(\mathbf{X}^{c_{\alpha}}\right) \prod_{z \in \mathcal{E}\left(\mathbf{X}^{c_{\alpha}}\right)}\left(w_{5}(z) w_{6}(z)-w_{3}(z) w_{4}(z)\right)
$$

where $\mathcal{P}_{\mathcal{E}}\left(\mathbf{X}^{c_{\alpha}}\right)$ is the product weight of $\mathbf{X}^{c_{\alpha}}$ with all the vertex weights associated with the vertices in $\mathcal{E}\left(\mathbf{X}^{c_{\alpha}}\right)$ divided out. This follows since $\prod_{z \in \mathcal{E}\left(\mathbf{X}^{c_{\alpha}}\right)}\left(w_{5}(z) w_{6}(z)-w_{3}(z) w_{4}(z)\right)$ allows for each vertex in $\mathcal{E}\left(\mathbf{X}^{c_{\alpha}}\right)$ to be a switch (i.e. weight $w_{3} w_{4}$ ) or an osculation (i.e. weight $\left.w_{5} w_{6}\right)$. The sign, $\epsilon_{\mathbf{X}}$ is correctly obtained since it is just -1 to the number of occurrences of a switch i.e. the number of factors of $-w_{3} w_{4}$. Thus we have the following:

$$
\begin{array}{r}
\operatorname{Det}\left\|\mathcal{P}\left(y_{\alpha}^{i} \rightarrow y_{\beta}^{f}\right)\right\|_{\alpha, \beta=1 \ldots N}=\sum_{\mathbf{X} \in \Omega} \epsilon_{\mathbf{X}} \mathcal{P}(\mathbf{X}) \\
=\sum_{\mathbf{X} \in \Omega-\Omega^{s}} \epsilon_{\mathbf{X}} \mathcal{P}(\mathbf{X})+\sum_{\mathbf{X} \in \Omega^{s}} \epsilon_{\mathbf{X}} \mathcal{P}(\mathbf{X})
\end{array}
$$

and since, by $(2.17)$ the $N$-walks in $\Omega-\Omega^{s}\left(=\Omega^{n} \cup \Omega^{e}\right)$ with any intersections cancel in pairs we get

$$
\begin{aligned}
& =\sum_{\mathbf{X} \in \Omega^{n}} \mathcal{W}(\mathbf{X})+\sum_{\mathbf{X} \in \Omega^{s}} \epsilon_{\mathbf{X}} \mathcal{P}(\mathbf{X}) \\
& =\sum_{\mathbf{X} \in \Omega^{n}} \mathcal{W}(\mathbf{X})+\sum_{\alpha \in \mathcal{I}} \sum_{\mathbf{X} \in \hat{\Omega}_{\alpha}^{s}} \epsilon_{\mathbf{X}} \mathcal{P}(\mathbf{X})
\end{aligned}
$$

where $\Omega^{n} \subset \Omega$ is the set of non-intersecting $N$-walks, using (2.18) gives,

$$
=\sum_{\mathbf{X} \in \Omega^{n}} \mathcal{W}(\mathbf{X})+\sum_{\alpha \in \mathcal{I}} \mathcal{P}_{\mathcal{E}}\left(\mathbf{X}^{c_{\alpha}}\right) \prod_{z \in \mathcal{E}\left(\mathbf{X}^{c_{\alpha}}\right)}\left(w_{5}(z) w_{6}(z)-w_{3}(z) w_{4}(z)\right)
$$

now, using the free fermion relation, 2.10 we get

$$
\begin{aligned}
& =\sum_{\mathbf{X} \in \Omega^{n}} \mathcal{W}(\mathbf{X})+\sum_{\alpha \in \mathcal{I}} \mathcal{P}_{\mathcal{E}}\left(\mathbf{X}^{c_{\alpha}}\right) \prod_{z \in \mathcal{E}\left(\mathbf{X}^{c_{\alpha}}\right)} w_{2}(z) \\
& =\sum_{\mathbf{X} \in \Omega^{n}} \mathcal{W}(\mathbf{X})+\sum_{\alpha \in \mathcal{I}} \mathcal{W}\left(\mathbf{X}^{c_{\alpha}}\right)
\end{aligned}
$$

and since all the canonical $N$-walks, $\mathbf{X}^{c_{\alpha}}$ are osculating we get

$$
=\sum_{\mathbf{X} \in \Omega^{*}} \mathcal{W}(\mathbf{X})
$$

as required. 


\section{Acknowledgements}

Financial support from the Australian Research Council is gratefully acknowledged by the authors.

\section{References}

[1] M. E. Fisher, J. Stat. Phys. 34, 667 (1984).

[2] P. J. Forrester, J. Phys. A. 22, L609 (1989).

[3] P. J. Forrester, J. Stat. Phys. 56, 767 (1989).

[4] P. J. Forrester, J. Phys. A. 23, 1259 (1990).

[5] P. J. Forrester, J. Phys. A. 24, 203 (1991).

[6] R. Brak, J. Essam, and A. L. Owczarek, Directed vesicles near an attractive wall, To appear in J. Stat. Phys., 1998.

[7] A. J. Guttmann, A. L. Owczarek, and X. G. Viennot, J. Phys. A. 31, 8123 (1998).

[8] I. M. Gessel and X. Viennot, Advances in Mathematics 58, 300 (1985).

[9] I. M. Gessel and X. Viennot, Determinants, paths, and plane partitions, preprint, 1989.

[10] B. Lindström, Bull. London. Math. Soc. 5, 85 (1973).

[11] S. Karlin and G. McGregor, Pacific Journal of Mathematics 9, 1141 (1959).

[12] F. Wu, Phys. Rev. 168, 539 (1968).

[13] R. Brak, J. Essam, and A. L. Owczarek, From the Bethe Ansatz to the Gessel-Viennot theorem, Submitted to Annals of Comb., 1998.

[14] R. Brak, An involution for the enumeration of osculating lattice paths, Preprint, 1999. 